\title{
Determinantes sociales, prácticas de alimentación y consecuencias nutricionales del parasitismo intestinal en niños de 7 a 18 meses de edad en Guapi, Cauca
}

\author{
Beatriz Eugenia Alvarado ${ }^{1}$, Luis Reinel Vásquez ${ }^{2}$ \\ ${ }^{1}$ Grupo AntroPacífico, Unidad de Epidemiología Clínica, Facultad de Ciencias de la Salud, Universidad del \\ Cauca, Popayán, Colombia. \\ ${ }^{2}$ Centro de Estudios en Microbiología y Parasitología, Facultad de Ciencias de la Salud, Universidad de I \\ Cauca, Popayán, Colombia.
}

Introducción. Los estudios de parasitismo intestinal en menores de 2 años raramente se realizan. Sus consecuencias nutricionales y la factibilidad de la prevención justifican su estudio. Objetivos. Identificar la prevalencia de parásitos intestinales patógenos, sus asociaciones con los determinantes sociales y las prácticas de alimentación y sus efectos sobre el estado nutricional.

Materiales y métodos. Se censó a los niños menores de 18 meses de una comunidad urbana de la región Pacífica colombiana. Se obtuvieron 136 muestras, que representaban $62 \%$ de la población de menores de 18 meses en el área de estudio. Se identificó la presencia de parásitos intestinales patógenos a partir del examen directo y el concentrado de Ritchie-Frick modificado. Se utilizaron regresiones logísticas múltiples para predecir la presencia de parásitos intestinales patógenos. Se calcularon los puntajes $Z$ de longitud para edad ( $\leq 2 \mathrm{DE}$, desnutrición crónica) y peso para longitud ( $\leq 2 \mathrm{DE}$, desnutrición aguda). Se realizaron análisis de covarianza para determinar la relación entre los parásitos intestinales patógenos y el estado nutricional con ajustes por edad.

Resultados. El 30,6\% de los niños estaban infectados: $26,2 \%$ presentaba helmintiasis (Ascaris lumbricoides, Trichuris trichiura, Strongyloides stercolaris); 14,9\% tenía protozoos (Giardia lamblia), y $11,8 \%$ poliparasitismo intestinal. La prevalencia de desnutrición aguda y crónica fue de $2,9 \%$ y $12,5 \%$, respectivamente. La ausencia de sanitario y una baja escolaridad de la madre se asociaron a una mayor presencia de poliparasitismo y helmintiasis $(p<0,05)$. La suspensión de la lactancia materna se asoció con la presencia de poliparasitismo (RM: 6,5 IC90\%: 1,9 a 21,5) y con la presencia de Giardia (RM: 2,89 IC90\%: 1,0 a 8,34). La desnutrición aguda fue más frecuente en niños infectados con $T$. trichiura y con varios parásitos $(p<0,05)$. Conclusión. Nuestros resultados sugieren que la desparasitación periódica debe incluir a la población lactante. La promoción de la lactancia materna es un elemento importante en la disminución de la carga de la enfermedad producida por los parásitos intestinales patógenos.

Palabras clave: parasitosis intestinales, lactante, estado nutricional, factores de riesgo, Colombia.

Social determinants, feeding practices and nutritional consequences of intestinal parasitism in young children

Introduction. Studies on intestinal intestinal parasitism in children under 2 years of age have rarely been performed. The nutritional consequences and feasibility of parasite prevention justify the current study.

Objectives. The study was undertaken with 3 goals: (1) to identify the prevalence of pathogenic intestinal parasites (PIP), (2) to determine the social factors, sanitary conditions and nutritional practices that predict PIP, and (3) to evaluate PIP effects on infant nutritional status.

Materials and methods. All mothers with children under 18 months of age and living in an urban community of coastal Colombia were invited to participate in the PIP study. A fecal sample was obtained from each of 136 children; they represented $62 \%$ of the total $7-18$ month- 
old population in the community. Presence of intestinal parasites was identified by direct microscopy and confirmed by a concentration test (Ritchie-Frick procedure). Logistic regressions were used to predict presence of PIP. The Z scores of length-for-age (<-2 SD-chronic malnutrition) and weight-for-length (<-2 SD-acute malnutrition) and covariance analysis were done to detect associations between intestinal parasitism and nutritional status.

Results. Of the 136 children, $30.6 \%$ were infected; $26.2 \%$ had helminth infections $(A$. lumbricoides, T. trichiura, S. stercoralis), $14.9 \%$ had protozoan infections (G. lamblia) and $11.8 \%$ had mixed infections. Wasting and stunting were present in $2.9 \%$ and $12.5 \%$, respectively. Lack of sanitary toilet facilities and low maternal education were related to mixed infections and presence of helminths $(p<0.05)$. Weaned children were at greater risk of mixed infections (Odds Ratio (OR) 6.5; 90\% Cl: 1.9-21.5) and of G. lamblia infections (OR: $2.89 ; 90 \% \mathrm{Cl}$ : 1.08.34). Children infected with $T$. trichiura and with mixed infections were more likely to show wasting $(p<0.05)$.

Conclusion. The high infections in young children indicate that they be included in periodic antiparasitic chemotherapy. Burden of disease associated to intestinal parasitism may be reduced if breastfeeding is continued beyond 6 months of age.

Keywords: intestinal diseases, parasitic, infant, nutritional status, risk factors, Colombia

Los casos de parasitismo intestinal en menores de 2 años representan 5 a $10 \%$ de los casos de parasitismo intestinal en el mundo. Sin embargo, son raros los estudios epidemiológicos publicados sobre este grupo de edad $(1,2)$. Los datos con que se cuenta en Colombia datan de la década de 1980 (3). Para este periodo se describieron prevalencias a nivel nacional de $12,8 \%$ de Ascaris lumbricoides, de 7,7\% de Trichuris trichiura y de $13,4 \%$ de Giardia lamblia en menores de un año, las cuales fueron mayores en niños de la costa Pacífica: $36,4 \%, 26,0 \%$ y $13,3 \%$, respectivamente (3). En los lactantes y los preescolares, la carga de la enfermedad producida por los parásitos intestinales se relaciona con efectos negativos en el crecimiento (4-6), el desarrollo motor y cognoscitivo (6) y la aparición de anemia (7). De allí que se hayan propuesto algunas estrategias como la desparasitación periódica en menores de dos años en recientes revisiones (2).

Las enfermedades infecciosas y la ingestión inadecuada de nutrientes constituyen las causas inmediatas de la desnutrición infantil (8). En efecto, el tratamiento para infecciones por $A$. lumbricoides y $T$. trichiura en niños menores de dos años ha mostrado beneficios en la ganancia de peso y en

\footnotetext{
Correspondencia:

Beatriz Eugenia Alvarado,1430 Boulevard Mont Royal, Outremont, Québec, Canada H2V 4P3

be.alvarado.llano@umontreal.ca
}

Recibido: 11/03/05; aceptado: 28/11/05 la disminución de la incidencia de desnutrición aguda (1). Excepto por algunos estudios longitudinales (9), las observaciones en niños menores de 2 años son contradictorias con respecto a los efectos de las helmintiasis en el crecimiento (10). En el caso de las infecciones por G. lamblia, los estudios tampoco son concluyentes, pero se han observado retardos lineales y ponderales en niños infectados (11-13). Los efectos de los parásitos intestinales patógenos sobre el estado nutricional infantil se han asociado con la disminución de la ingestión de nutrientes, sea por anorexia, disminución de su absorción o aumento de su utilización $(4,5)$.

El parasitismo intestinal infantil está, a su vez, determinado por el acceso de las poblaciones a recursos materiales (posesión de bienes, calidad de la vivienda), recursos humanos (educación) y de saneamiento (tipo de sanitario, fuente de consumo de agua), así como a las prácticas de cuidado materno (alimentación, prevención e higiene); puede considerarse como un mecanismo intermedio entre estos factores y el estado nutricional $(14,15)$ (figura 1). En efecto, las parasitosis intestinales en los menores de dos años se encuentra en mayor proporción en donde predominan la pobreza, las inadecuadas condiciones sanitarias y la mala calidad de la vivienda (1619). Aunque con menor frecuencia, también se ha establecido que las prácticas de cuidado materno en cuanto a la alimentación se relacionan con la frecuencia de parasitosis intestinal, que, a 
través de sus efectos sobre estas infecciones, afecta el estado nutricional infantil (figura 1). Por ejemplo, la lactancia materna después de los 6 meses de edad se relaciona con la disminución de la frecuencia de giardiosis y de helmintiasis $(20,21)$.

Siguiendo la propuesta de la figura 1, este trabajo tiene como objetivos identificar la frecuencia de parásitos intestinales patógenos, los recursos materiales, humanos y ambientales, y las prácticas de cuidado (alimentarias), así como las consecuencias sobre el estado nutricional infantil de las parasitosis intestinales patógenas en menores de 18 meses de edad de una comunidad del Pacífico colombiano. Los resultados de este estudio se proponen como una evidencia adicional para realizar intervenciones que disminuyan la frecuencia de parásitos intestinales en los menores de 2 años.

\section{Materiales y métodos}

\section{Diseño y área de estudio}

Este estudio transversal se realizó en la cabecera urbana del municipio de Guapi, Costa Pacífica caucana, y se planteó como objetivo conocer los determinantes sociales y los factores biológicos asociados al estado nutricional en niños de 6 a 18 meses (22). La población del municipio se calcula en 30.000 habitantes, 18.000 de ellos en el casco urbano del municipio. Guapi representa fielmente las condiciones sociales descritas para otras comunidades de la Costa Pacífica: baja disponibilidad de servicios sanitarios, precarias condiciones de vivienda, hacinamiento, aislamiento geográfico de centros urbanos $(23,24)$. La cabecera urbana cuenta con un hospital local de nivel básico de atención (consulta, urgencias primarias y programas de prevención y promoción) y dos puestos de atención privados. Las

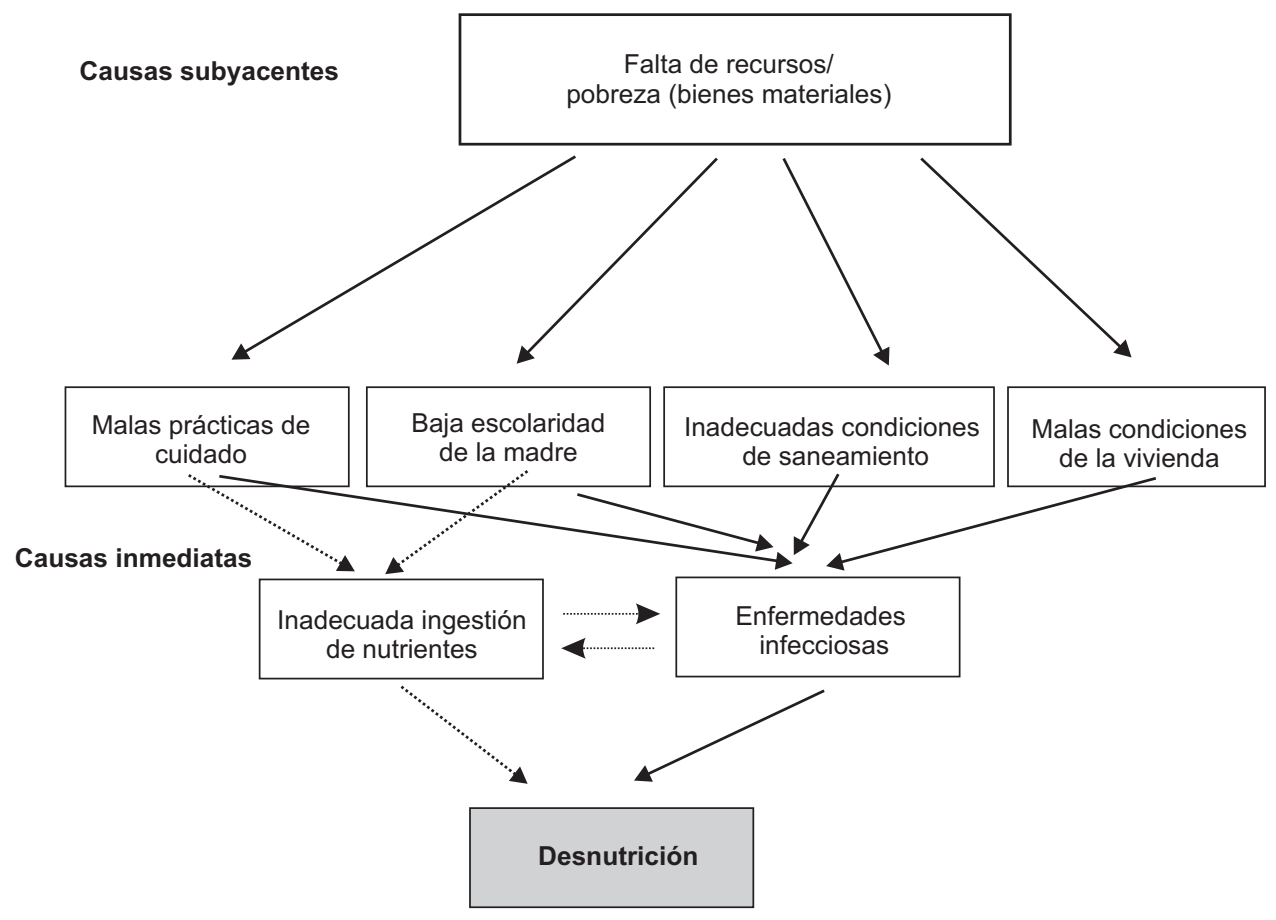

Figura 1. Causas de desnutrición infantil. La gráfica presenta dos niveles de causas. El primero, el subyacente, está compuesto principalmente por los determinantes sociales y las prácticas de cuidado infantil. El segundo nivel, el de las causas inmediatas, está compuesto por la ración dietética y las enfermedades infecciosas. Las líneas punteadas corresponden a las asociaciones no evaluadas en nuestros análisis. Traducida al español Baqui y Black (referencia 8), y reproducida con autorización de Lippincott Williams \& Wilkins. 
campañas de desparasitación masiva no se presentan en esta población y la mayoría de las personas de estas comunidades recurren a la medicina tradicional y a las plantas medicinales ante la presencia de parásitos (25).

\section{Población de estudio}

Un censo del total de madres o cuidadores primarios de niños entre los 6 y 18 meses de edad se realizó en los 20 barrios de la cabecera urbana. Se encontraron 216 madres de las 246 censadas en la primera visita, 193 de las cuales aceptaron participar y tenían los datos completos. Las madres que se incluyeron en este estudio fueron aquéllas cuyos niños tenían entre 7 y 18 meses (163/193). No se tomó muestra de materia fecal a las madres de niños de 6 meses porque fueron incluidas en un estudio longitudinal, en el cual la frecuencia de parásitos se midió al final del seguimiento. De las madres que llenaban este último criterio de inclusión, 136 entregaron muestras de materia fecal (83\%). Los cálculos del tamaño de muestra se obtuvieron para determinar la asociación entre escolaridad de la madre y estado nutricional (razón de momios: 2,0 ); 300 niños constituían la muestra para un nivel de confianza de $90 \%$ y poder de $80 \%$ (26).

\section{Recolección de datos}

La información del estudio se recolectó entre marzo y octubre de 2002. Antes de iniciar el estudio se ajustó una encuesta estructurada dividida en 7 secciones. Se realizaron dos visitas a cada una de las madres; en la primera se recogía información sobre las características demográficas, condiciones de vida y salud materno-infantil. En la segunda visita se interrogaba sobre las prácticas de alimentación y se realizaba la medición de la longitud y el peso del niño. Las entrevistas fueron realizadas por mujeres con formación escolar (primera visita) y por auxiliares de enfermería que vivían en la región (segunda visita), entrenadas antes del inicio del estudio. Siguiendo el modelo presentado en la figura 1, nuestros análisis incluyeron las variables relacionadas con los cuatro parámetros estudiados: la posesión de bienes materiales, la educación de la madre, la calidad de la vivienda y las prácticas de alimentación.
El índice de posesiones materiales (nevera, estufa, radio, teléfono y electricidad; rango de 0 a 5) y la calidad de la vivienda (tipo de piso) se consideraron como recursos materiales, mientras que la escolaridad de la madre (años de estudio) se consideró como recurso humano. Dada la homogeneidad de esta población en cuanto a la fuente de agua, el tipo de sanitario se consideró como indicador de las condiciones sanitarias de la vivienda. Entre las prácticas de alimentación se determinaron: 1) la práctica de alimentación predominante en los primeros 6 meses de vida, siguiendo la definición propuesta por Piwoz y col. (27) (anexo 1); 2) el primer alimento introducido (sólidos, cereales no fortificados, cereales fortificados o líquidos no lácteos); 3) la edad de inicio de la alimentación complementaria (cualquier alimento o líquido diferente a la leche materna, leche de fórmula o artificial); 4) el uso de biberón, y 5) la práctica de lactancia al momento del estudio (ausencia, lactancia en menores y mayores de 12 meses).

\section{Medición de longitud y peso}

La longitud se midió en posición decúbito en todos los niños. En todos los niños de 6 a 18 meses se utilizó un medidor de talla de madera con soporte en la cabeza y una base móvil con freno en el contacto de los pies (28). La medición de la longitud fue hecha por uno de los investigadores y por dos auxiliares de enfermería previamente entrenadas. Se tomaron cuatro medidas en la mayoría de los niños. El promedio de las cuatro medidas se empleó para el cálculo de los puntajes Z. El peso del niño se midió con una balanza suspendida (Detecto, $25 \mathrm{~kg} / 500 \mathrm{~g}$ ). La misma balanza fue utilizada en todos los niños y calibrada antes de cada toma. El niño se pesó completamente desnudo en dos oportunidades (28). El promedio de las dos medidas se utilizó para los análisis. Se hizo la conversión de la longitud y el peso del niño a puntajes Z (unidades de desviación estándar) según la población de referencia del año 1978 de la Organización Mundial de la Salud (29). Se calcularon los puntajes $Z$ de peso para longitud como medida de déficit a corto plazo (crecimiento de masa muscular y grasa del niño), y de longitud para edad como medida de déficit a largo plazo (crecimiento esquelético). Los valores de Z 
menores o iguales a -2 desviaciones entandar se emplearon como puntos de corte para definir la desnutrición aguda en el caso de peso para longitud, y de desnutrición crónica en el caso de longitud para edad (30).

\section{Medición del parasitismo intestinal}

Se obtuvo una muestra de materia fecal de los niños participantes. La muestra fue procesada dentro de las primeras 24 horas en formol al 5\% para su transporte aéreo al Laboratorio del Centro de Estudios en Microbiología y Parasitología (CEMPA). Todas las muestras fueron leídas por un parasitólogo experimentado. Se realizó un examen directo de las muestras siguiendo las recomendaciones de la Organización Mundial de la Salud (31). Posteriormente, se realizó la lectura de las muestras por el método de concentración de Ritchie-Frick modificado (32). El conteo de huevos se realizó mediante la técnica de KatoKatz modificada (33). Se definió parasitismo intestinal como la presencia de una muestra positiva con cualquiera de los dos métodos.

\section{Análisis}

Se analizaron los resultados de 136 niños con muestra de materia fecal, empleando el programa SPSS 10.0. Se establecieron las diferencias de proporciones y regresiones logísticas múltiples para establecer los factores asociados a la presencia de parásitos intestinales. Se calcularon la razón de momios ( $\mathrm{RM})$ y los intervalos de confianza de $90 \%$ para cada una de las variables de predicción. Se estableció un nivel de confianza de $90 \%$ de acuerdo con la recomendación de Rothman y Greenland para tamaños de muestra pequeños (34). Conservando la estructura jerárquica de los datos, se consideraron como factores de confusión en la asociación entre las prácticas de alimentación y la presencia de parásitos intestinales, la posesión de bienes, la educación de la madre y las condiciones sanitarias (35). Los datos antropométricos presentaron una distribución normal y fueron tratados de manera continua en nuestros análisis. Se establecieron modelos de análisis de covarianza (ANCOVA), para evaluar las diferencias en el estado nutricional de acuerdo con la presencia de parásitos intestinales patógenos y ajustando por la edad del niño.

\section{Consideraciones éticas}

El protocolo del estudio fue aprobado por el Comité de Ética de la Universidad de Montreal y por el Comité de Ética de la Organización Panamericana de la Salud. Se envió una invitación para la participación en el estudio y se obtuvo el consentimiento informado de las madres de los niños antes de iniciar el estudio. Las madres de los niños positivos para parásitos intestinales fueron informadas y se distribuyó el tratamiento correspondiente.

\section{Resultados}

La población de estudio incluyó 136 madres con promedio de escolaridad de 6,6 años; de ellas, $8,6 \%$ no había asistido a la escuela. El $66 \%$ de las madres no tenía acceso a sanitario y el $71 \%$ vivía en hogares con piso de madera o tierra. En las prácticas de alimentación primaba la lactancia parcial en los primeros 6 meses de vida $(90,4 \%)$, mientras que la lactancia exclusiva/predominante y la suspensión de la lactancia fueron raras, 4,4\% y $5,1 \%$, respectivamente. El $73 \%$ de las madres reportó haber lactado a su niño en las últimas 24 horas. El tiempo promedio de introducción de la alimentación complementaria fue de 3 meses; las legumbres, tubérculos y cereales no fortificados son los primeros alimentos introducidos. El uso de biberón era frecuente en la población de estudio con $69,9 \%$.

El promedio de edad de los niños fue de 12,26 meses (DE=3,51), 86 de ellos niños y 77 niñas. El 30,6\% de los niños estaba infectado con, al menos, un parásito intestinal patógeno: $26,2 \%$ con helmintos ( $A$. lumbricoides, $T$. trichiura, $S$. stercoralis) y $14,9 \%$ con protozoos (G. lamblia). Las infecciones con más de un parásito se presentaron en $11,8 \%$ de los niños; las más frecuentes fueron las causadas por $A$. lumbricoides y $T$. trichiura, con $9,5 \%$. Dos niños fueron positivos para $S$. stercoralis. Siete niños presentaron parásitos intestinales no patógenos (Endolimax nana, Entamoeba coli y Blastocystis hominis). El promedio de huevos por gramo para A. lumbricoides fue de 480 (DE= 555; rango: 5 a 2500), y para T. trichiura de 323 ( $D E=439$; rango: 3 a 1.817). La frecuencia de parásitos intestinales patógenos aumentó con la edad, con prevalencias 
del orden del $40 \%$ en los mayores de 15 meses (figura 2).

\section{Predictores de parasitismo intestinal}

La edad del niño se asoció de manera positiva con la presencia de poliparasitismo intestinal $\mathrm{RM}=$ 1,22; intervalo de confianza: $90 \%$ (IC90\%): 1,07 a 1,40$)$ y de helmintos $(R M=1,14 ;$ IC90\%: 1,04 a $1,26)$, pero no con la presencia de protozoos patógenos (RM=1,09; IC90\%: 0,97 a 1,22). Los niños tuvieron mayor riesgo de presentar $G$. lamblia que las niñas ( $\mathrm{RM}=3,66 \mathrm{IC} 90 \%$ : 1,27 a 10,55). El bajo acceso a recursos materiales, el bajo nivel de educación de la madre, así como la falta de sanitario y la vivienda construida en madera o tierra resultaron predictores de mayor proporción de poliparasitismo, infección por $A$. lumbricoides o T. trichiura y presencia de G. lamblia (cuadro 1). La probabilidad de tener poliparasitismo intestinal fue 4 veces mayor si la madre no había completado la primaria (RM=3,9 IC90\%: 1,4 a $10,6)$, o si la vivienda no tenía sanitario ( $\mathrm{RM}=3,9$ IC90\%: 1,1 a 14,1), y de casi 7 veces si en la vivienda el suelo era de madera o tierra $(\mathrm{RM}=6,9$ IC90\%: 1,2 a 39,1). La infección con helmintos fue también más frecuente entre los niños con desventajas materiales, con madres con menos años de primaria y que vivían en malas condiciones sanitarias. En cuanto a la frecuencia de giardiasis, especialmente las condiciones sanitarias inadecuadas y el tipo de suelo se asociaron con una probabilidad 11 veces mayor

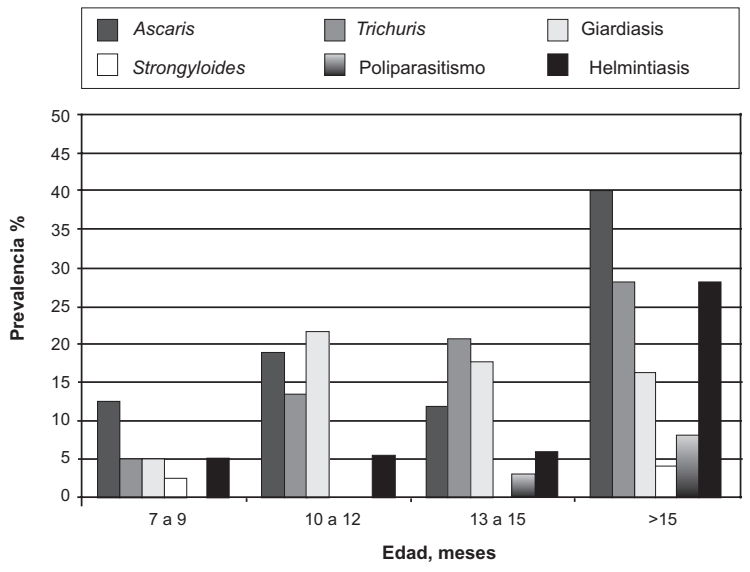

Figura 2. Prevalencia de parasitismo intestinal por grupos de edad. de infección (RM=11,6 IC90\%: 2,1 a 64,5) y 4 veces mayor ( $R M=4,2 \mathrm{IC} 90 \%: 1,1$ a 14,9).

Los niños que aún recibían leche materna en el momento de la encuesta presentaron una menor proporción de poliparasitismo intestinal $(p=0,01)$, frecuencia de helmintos $(p=0,01)$ y de giardiasis $(p=0,05)$. Los cálculos de razones de Momios muestran que los niños amamantados después de los 6 meses de edad tenían menor probabilidad de presentar poliparasitismo e infección por protozoos (cuadros 1 y 2). Los niños con lactancia materna de manera exclusiva o predominante en los primeros 6 meses de vida no registraron presencia de parásitos intestinales patógenos comparados con aquellos con lactancia materna de manera parcial y aquéllos que suspendieron la lactancia antes de los 6 meses ( $p=0,10$ para poliparasitismo; $p=0,06$ para helmintiasis). Entre los niños que habían recibido lactancia materna parcialmente o que suspendieron la lactancia materna en los primeros 6 meses de vida, el tiempo de introducción de los alimentos complementarios no se asoció de manera significativa con la presencia de parásitos intestinales patógenos (cuadros 1 y 2). El uso de biberón y el tipo de alimento introducido tampoco se asociaron con la presencia de parásitos intestinales $(p>0,20$ para todas las variables dependientes).

\section{Estado nutricional y parásitos intestinales}

El promedio de puntaje Z para los dos indicadores antropométricos estuvo por debajo del promedio de la población de referencia (cuadro 3). La prevalencia de desnutrición aguda ( $\leq 2 \mathrm{DE}$ de peso para longitud) y crónica ( $\leq 2 \mathrm{DE}$ de longitud para edad) fue de $2,9 \%$ y $12,5 \%$, respectivamente. La prevalencia de desnutrición aguda fue mayor después de los 12 meses, sin diferencias entre los niños y las niñas ( $p>0,20$, datos no mostrados). La prevalencia de baja longitud para edad fue mayor después de los 12 meses de edad, siendo la proporción mayor en los niños que en las niñas, con promedios en el puntaje $Z$ significativamente mayores para las niñas $(p=0,02$, datos no mostrados). La presencia de parásitos intestinales patógenos se relacionó con menores puntajes $Z$ en los dos indicadores; sin embargo, las 
Cuadro 1. Razón de momios calculada a partir de modelos de regresión logísticos.

\begin{tabular}{|c|c|c|c|c|c|}
\hline \multirow[t]{2}{*}{ Variables predictoras } & \multirow[t]{2}{*}{ Total $(\%)^{1}$} & \multicolumn{2}{|c|}{ Poliparasitismo ${ }^{2}$} & \multicolumn{2}{|c|}{ Helmintiasis ${ }^{3}$} \\
\hline & & $\%^{a}$ & RM (IC90\%) & $\%$ & RM (IC90\%) \\
\hline \multicolumn{6}{|l|}{ Escolaridad de la madre } \\
\hline $0-5$ años & $64(47,1)$ & 18,8 & $3,90(1,45-10,62)$ & 19,4 & $2,37(1,20-4,67)$ \\
\hline Más de 5 años & $72(52,9)$ & 5,6 & 1,00 & 34,4 & 1,00 \\
\hline$p$ & & 0,01 & 0,02 & 0,05 & 0,05 \\
\hline \multicolumn{6}{|c|}{ Índice de posesiones materiales } \\
\hline $0 / 1$ & $45(33,1)$ & 17,8 & $2,63(0,65-10,65)$ & 35,6 & $4,57(1,45-14,3)$ \\
\hline$p$ & & & 0,21 & & 0,02 \\
\hline $2 / 3$ & $63(46,3)$ & 9,5 & $1,36(0,33-5,65)$ & 27,0 & $3,17(1,03-9,70)$ \\
\hline$p$ & & & 0,71 & & 0,09 \\
\hline 4 a 6 & $28(20,6)$ & 7,1 & 1,00 & 10,7 & 1,00 \\
\hline$p$ & & 0,25 & & 0,06 & \\
\hline \multicolumn{6}{|l|}{ Tipo de sanitario } \\
\hline Pozo séptico/letrina/nada & $91(66,9)$ & 15,4 & $3,90(1,08-14,09)$ & 34,1 & $4,00(1,66-9,43)$ \\
\hline Acueducto & $45(33,1)$ & 4,4 & 1,00 & 11,1 & 1,00 \\
\hline$p$ & & 0,06 & 0,07 & 0,004 & 0,006 \\
\hline \multicolumn{6}{|l|}{ Tipo de suelo } \\
\hline Madera/tierra & $97(71,3)$ & 15,0 & $6,94(1,23-39,16)$ & 32,0 & $3,19(1,32-7,70)$ \\
\hline Baldosa/cemento & $39(28,7)$ & 2,6 & 1,00 & 12,8 & 1,00 \\
\hline$p$ & & 0,03 & 0,06 & 0,02 & 0,06 \\
\hline \multicolumn{6}{|c|}{ Lactancia en los primeros 6 meses $^{a}$} \\
\hline Exclusiva/predominante & $6(4,4)$ & 0,0 & 0,0 (0,0-inf) & 0,0 & 0,0 (0,0-inf) \\
\hline$p$ & & & 0,77 & & 0,73 \\
\hline Parcial & $123(90,4)$ & 11,1 & $0,40(0,07-2,90)$ & 26,0 & $0,36(0,09-1,42)$ \\
\hline$p$ & & & 0,36 & & 0,17 \\
\hline Suspendida & $7(5,1)$ & 28,6 & 1,00 & 57,1 & 1,00 \\
\hline$p$ & & 0,25 & & 0,06 & \\
\hline \multicolumn{6}{|l|}{ Lactancia actual $^{\mathrm{a}}$} \\
\hline$p$ & & & 0,009 & & 0,50 \\
\hline Sí - menor de 12 meses & $41(30,1)$ & 5,2 & $1,65(0,04-6,83)$ & 24,4 & $0,98(0,30-3,23)$ \\
\hline$p$ & & & 0,55 & & 0,90 \\
\hline Sí - mayores de 12 meses & $58(42,6)$ & 7,3 & 1,00 & 17,2 & 1,00 \\
\hline$p$ & & 0,003 & & 0,01 & \\
\hline \multicolumn{6}{|l|}{$\begin{array}{l}\text { Inicio de alimentación } \\
\text { complementaria } a, b\end{array}$} \\
\hline Antes de 4 meses & $72(52,9)$ & 15,6 & $2,52(0,93-6,79)$ & 30,6 & $1,18(0,59-2,35)$ \\
\hline Después de 4 meses & $64(47,1)$ & 8,3 & 1,00 & 21,9 & 1,00 \\
\hline$p$ & & 0,18 & 0,19 & 0,25 & 0,45 \\
\hline
\end{tabular}

Parasitismo intestinal explicado por determinantes sociales y prácticas de alimentación.

${ }^{1}$ Frecuencia de la variable de predicción en la población de estudio.

2 La positividad representa la presencia de 2 y 3 parásitos.

${ }^{3}$ La positividad representa la presencia de Ascaris o Trichuris.

a RM ajustadas por educación, condiciones materiales y sanitarias.

${ }^{b} \mathrm{RM}$ calculadas para aquellos niños que no recibieron lactancia materna de manera exclusiva o predominante hasta los 6 meses. 
diferencias fueron significativas para la presencia de infección por $T$. trichiura y el puntaje $Z$ de peso para longitud (cuadro 3). La prevalencia de desnutrición aguda fue mayor en los niños con

Cuadro 2. Razón de momios calculada a partir de modelos de regresión logísticos. Giardiasis explicada por determinantes sociales y prácticas de alimentación.

\begin{tabular}{|c|c|c|}
\hline \multirow[t]{2}{*}{ Variables predictoras } & \multicolumn{2}{|r|}{ Giardiasis ${ }^{1}$} \\
\hline & $\%$ & RM (IC90\%) \\
\hline \multicolumn{3}{|l|}{ Escolaridad de la madre } \\
\hline 0- 5 años & 16,7 & $0,71(0,32-1,61)$ \\
\hline Más de 5 años & 12,5 & 1,00 \\
\hline$p$ & 0,49 & 0,49 \\
\hline \multicolumn{3}{|l|}{$\begin{array}{l}\text { Índice de posesiones } \\
\text { materiales }\end{array}$} \\
\hline $0 / 1$ & 17,8 & $1,29(0,43-3,88)$ \\
\hline$p$ & & 0,77 \\
\hline $2 / 3$ & 12,7 & $0,87(0,29-2,58)$ \\
\hline$p$ & & 0,82 \\
\hline 4 a 6 & 14,3 & 1,00 \\
\hline$p$ & 0,76 & \\
\hline \multicolumn{3}{|l|}{ Tipo de sanitario } \\
\hline Pozo séptico/letrina/nada & 20,9 & $11,60(2,08-64,54)$ \\
\hline Acueducto & 2,2 & 1,00 \\
\hline$p$ & 0,004 & 0,02 \\
\hline \multicolumn{3}{|l|}{ Tipo de suelo } \\
\hline Madera/tierra & 18,6 & $4,21(1,18-14,96)$ \\
\hline Baldosa/cemento & 5,1 & 1,00 \\
\hline$p$ & 0,04 & 0,06 \\
\hline \multicolumn{3}{|c|}{ Lactancia primeros 6 meses $^{a}$} \\
\hline Exclusiva/predominante & 16,7 & $0,62(0,05-7,68)$ \\
\hline$p$ & & 0,76 \\
\hline Parcial & 13,8 & $0,34(0,06-1,78)$ \\
\hline$p$ & & 0,28 \\
\hline Suspendida & 28,6 & 1,00 \\
\hline$p$ & 0,55 & \\
\hline \multicolumn{3}{|l|}{ Lactancia actuala } \\
\hline No & 24,3 & $2,89(1,00-8,34)$ \\
\hline$p$ & & 0,09 \\
\hline Sí - menor de 12 meses & 8,6 & $1,39(0,45-4,23)$ \\
\hline$p$ & & 0,62 \\
\hline Sí - mayores de 12 meses & 14,6 & 1,00 \\
\hline$p$ & 0,10 & \\
\hline \multicolumn{3}{|l|}{$\begin{array}{l}\text { Inicio de alimentación } \\
\text { complementaria } a, b\end{array}$} \\
\hline Antes de 4 meses & 18,1 & $1,60(0.66-3.91)$ \\
\hline Después de 4 meses & 10,9 & 1,00 \\
\hline$p$ & 0,24 & 0,38 \\
\hline
\end{tabular}

1. La positividad representa la presencia de giardiosis.

a. RM ajustadas por educación, condiciones materiales y sanitarias.

b. RM calculadas para aquellos niños que no recibieron lactancia materna de manera exclusiva o predominante hasta los 6 meses.
T. trichiura y en niños con poliparasitismo, en quienes la prevalencia alcanzó el 12,5\% (cuadro 3). $\mathrm{Ni}$ los puntajes $Z$ de longitud para la edad ni la proporción de desnutrición crónica fueron diferentes entre los niños parasitados y no parasitados en nuestra población de estudio.

\section{Discusión}

La presencia de parásitos intestinales patógenos en poblaciones deprimidas socialmente como la de Guapi comienza a temprana edad con prevalencias del orden de $30 \%$ en niños menores de 18 meses. A los 7 meses de edad, el $10 \%$ de los niños ya está infectado. La frecuencia de helmintos en nuestra población de lactantes, $20 \%$, contrasta con la de los lactantes en áreas urbanizadas $(0 \%)$, donde predominan los protozoos, 44\% (36), y son similares a las encontradas en lactantes de áreas rurales de la Costa Pacífica, 37\% (37). Las condiciones sanitarias y sociales de la región de estudio están entre las más bajas de Colombia $(23,24)$; ello podría explicar que la situación de morbilidad por parásitos continúe casi inmodificable después de 20 años (3) .

Como lo evidencian otros estudios, el parasitismo intestinal en los menores de dos años es el reflejo de las condiciones materiales, el acceso a servicios sanitarios y la educación de la madre $(16-19,38)$. La escolaridad de la madre en particular, determinante éste importante de comportamientos adecuados de higiene, mejores prácticas de cuidado del niño y mejores conocimientos en salud $(39,40)$, se asocia en nuestro estudio con la presencia de parásitos intestinales patógenos (18). Así, las madres con menos años de primaria tienen mayor probabilidad de que sus hijos tengan poliparasitismo y algún tipo de helminto. De igual manera se ha señalado que la falta de acceso a un sanitario y las condiciones materiales inadecuadas de la vivienda aumentan la probabilidad de cualquier tipo de infección por parásitos intestinales patógenos entre la población infantil (41-43).

El aporte más importante de este estudio se relaciona con el efecto de las prácticas de alimentación en la frecuencia de parásitos intestinales patógenos. En particular, la lactancia 
Cuadro 3. ANCOVA (análisis de covarianza) y ji² para los indicadores antropométricos por infección parasitaria.

\begin{tabular}{|c|c|c|c|c|c|c|}
\hline \multirow[t]{2}{*}{ Parásitos intestinales } & \multicolumn{3}{|c|}{ Índice $\mathrm{Z}$ de longitud/edad ${ }^{1}$} & \multicolumn{3}{|c|}{ Puntaje $Z$ de peso/longitud ${ }^{1}$} \\
\hline & Media (EE) & $\%{ }^{a}$ & $\%^{b}$ & Media (EE) & $\%{ }^{a}$ & $\%^{b}$ \\
\hline A. lumbricoides (negativo) & $-0,65(0,10)$ & 12,7 & 20,9 & $-0,29(0,09)$ & 2,7 & 21,8 \\
\hline A. lumbricoides (positivo) & $-0,74(0,21)$ & 11,5 & 30,8 & $-0,24(0,19)$ & 3,8 & 19,2 \\
\hline Valor $p$ & 0,71 & 0,55 & 0,83 & 0,92 & & \\
\hline T. trichiura (negativo) & $-0,64(0,10)$ & 12,2 & 20,0 & $-0,21(0,08)$ & 0,9 & 20,9 \\
\hline T. trichiura (positivo) & $-0,84(0,24)$ & 14,3 & 38,1 & $-0,68(0,21)$ & 14,3 & 23,8 \\
\hline Valor $p$ & 0,43 & 0,15 & 0,04 & 0,003 & & \\
\hline G. lamblia (negativa) & $-0,64(0,10)$ & 12,1 & 20,7 & $-0,28(0,08)$ & 2,6 & 22,4 \\
\hline G. lamblia (positiva) & $-0,85(0,24)$ & 15,0 & 35,0 & $-0,26(0,21)$ & 5,0 & 15,0 \\
\hline Valor $p$ & 0,41 & 0,29 & 0,92 & 0,65 & & \\
\hline Poliparasitismo (ausente) & $-0,55(0,11)$ & 11,6 & 16,3 & $-0,16(0,10)$ & 1,2 & 18,6 \\
\hline Poliparasitismo (1 PI) & $-0,94(0,18)$ & 14,7 & 32,4 & $-0,51(0,16)$ & 2,9 & 32,4 \\
\hline Poliparasitismo (2 y $3 \mathrm{PI})$ & $-0,73(0,27)$ & 12,5 & 37,5 & $-0,40(0,24)$ & 12,5 & 12,5 \\
\hline Valor $p$ & 0,18 & 0,15 & 0,18 & 0,05 & & \\
\hline Total & $-0,68(1,05)$ & 12,5 & 22,8 & $-0,29(1,05)$ & 2,9 & 21,3 \\
\hline
\end{tabular}

a. Prevalencia de desnutrición: puntaje $Z$ menor de $-2 \mathrm{DE}$.

b. Porcentaje de niños con puntaje $Z$ entre -2 DE y -1 DE.

1. Todas las medias están ajustadas por la edad del niño.

materna después de los 6 meses de edad se relaciona con una menor proporción de poliparasitismo intestinal, menor infección por $A$. lumbricoides o T. trichiura, y menor frecuencia de G. lamblia. A la lactancia materna se le atribuyen efectos antinfecciosos como la disminución de los episodios de enfermedades gastrointestinales, de neumonía y de otitis media aguda (44). Los estudios como el nuestro añaden evidencia sobre la relación entre la lactancia materna y la presencia de parásitos intestinales patógenos. En el caso de las helmintiasis, un estudio de 400 niños africanos mostró que los niños con lactancia materna de manera exclusiva o parcial, comparados con los que habían suspendido la lactancia, tenían menor excreción de helmintos, y que esta asociación no guardaba relación con la frecuencia de parásitos intestinales patógenos en las madres (45). En un estudio transversal en Brasil, los niños menores de 2 años destetados presentaron más enteroparásitos que los niños que recibían leche materna (21). Los estudios de laboratorio muestran que la leche materna contiene anticuerpos para G. lamblia (46). Los estudios en niños menores de 2 años han estimado que la lactancia materna disminuye los episodios sintomáticos de giardiasis (20), mientras que los estudios de cohorte muestran que el pico de infección por G. lamblia coincide con el inicio de la alimentación complementaria (12).

Otro hallazgo que respalda la hipótesis anterior es la observación en Guapi de un aumento significativo de la frecuencia de parásitos intestinales patógenos con la edad, que se corresponde con el patrón de destete (Alvarado et al., en impresión). En uno de los pocos estudios en poblaciones de lactantes se muestra que la infección por $A$. lumbricoides incrementa a partir de los 6 meses y se presenta con mayor frecuencia después de los 12 meses, patrón similar al encontrado en los niños de Guapi (47). Según los autores, los anticuerpos maternos protegen de las infecciones parasitarias a los niños durante los primeros seis meses de vida, mientras que la exposición del niño a las condiciones ambientales, y posiblemente el destete (no evaluado), explican el aumento con la edad.

El efecto de los parásitos intestinales sobre el estado nutricional en esta población puede considerarse marginal, a pesar de la alta prevalencia de parásitos intestinales patógenos. Nuestros resultados muestran que sólo las infecciones por helmintos, especialmente la infección por $T$. trichiura, parecen estar relacionadas con menores promedios en el 
indicador de peso para longitud y mayor frecuencia de desnutrición aguda, como se ha reportado en los niños no tratados en estudios experimentales con antihelmínticos $(1,48-49)$. Las infecciones con T. trichiura producen en los niños alteraciones en la mucosa intestinal, disminución del consumo de alimentos, deficiencia de hierro y pérdida de proteínas (5). Los menores promedios en el indicador de peso para longitud entre los infectados con este parásito se pueden explicar por un episodio agudo con disminución de peso asociado a anorexia o diarrea causada por el parásito (50). En cuanto a la asociación con la desnutrición crónica, dado que ésta no ocurre sino tras largos periodos de exposición a fallas en la ganancia de peso (51), es posible que se deba a los efectos crónicos de la tricocefalosis en la mala absorción de proteínas y hierro (50).

La ausencia de asociación con el indicador de longitud para edad (crecimiento lineal), se podría explicar por el hecho de que en nuestra población, las infecciones por áscaris y tricocéfalos no fueron graves. En efecto, el conteo de huevos en el $100 \%$ de los niños fue menor de 5.000 para $A$. lumbricoides y menor de 1.000 para $T$. trichiura. Recientemente se ha sugerido que los efectos de la infección por A. lumbricoides sobre el estado nutricional en poblaciones infantiles está asociada a la presentación de infecciones graves (5); en otros estudios se ha observado que estas asociaciones son más importantes en los mayores de 2 años (48). Igualmente, en ensayos comunitarios con antihelmínticos se ha evidenciado que la falta de mejoría en el estado nutricional entre los niños intervenidos puede deberse a que las infecciones en ellos son leves (49).

Algunos autores sostienen que la evidencia de la asociación entre la infección por G. lamblia y el estado nutricional no es concluyente (11). En algunos estudios en menores de 2 años, no se ha observado asociación entre las infecciones por G. lamblia y el estado nutricional (49), mientras que otros sólo han evidenciado una asociación con la perdida de peso $(12,13)$, y otros con un retardo del crecimiento lineal (11). Las diferencias entre los estudios pueden deberse al carácter transversal de las asociaciones (la desnutrición puede predisponer a la presencia de infección), a las diferencias en la presentación clínica de diferentes cepas de giardiasis, o a las diferencias en la distribución de los factores de riesgo. Por ejemplo, en los estudios en los que se ha encontrado asociación de la giardiasis con el estado nutricional infantil, los episodios de giardiasis se caracterizaban por diarrea persistente (52). En nuestro estudio no se encontraron diferencias entre la presencia de diarrea en los 15 días previos o las hospitalizaciones por diarrea y la presencia de G. lamblia. La otra razón para estas diferencias podría encontrarse en la hipótesis del efecto de la lactancia materna propuesta por nuestro equipo y por otros autores (12), según la cual, la lactancia podría mitigar los efectos negativos de las infecciones por protozoos en poblaciones donde la duración de la lactancia materna es prolongada, como ocurre en la del presente estudio (11 meses).

El tamaño de la muestra, los sesgos de clasificación y las muestras incompletas pueden afectar los resultados. Nuestro tamaño de muestra es insuficiente para explicar algunas asociaciones. El número de niños con lactancia materna de manera exclusiva y predominante es muy bajo (6 niños) para permitir conclusiones en torno a la falta de efectos sobre la presencia de parásitos intestinales patógenos. El poder estadístico necesario para detectar asociaciones entre parásitos intestinales patógenos y desnutrición infantil con base en los datos encontrados sería del orden del $35 \%$. Sin embargo, el tamaño de la muestra es suficiente para respaldar las hipótesis relacionadas con los factores sociales, ambientales y con algunas prácticas de alimentación como la lactancia materna después de los 6 meses de edad. Se obtuvo una sola muestra de materia fecal directo, lo cual disminuye la sensibilidad del examen de materia fecal directo. Sin embargo, los resultados del examen directo fueron complementados con el concentrado de Ritchie-Frick (RF), con lo que se pudo aumentar la sensibilidad de la prueba (32). Finalmente, no se obtuvieron muestras de materia fecal en $16 \%$ de los niños hijos de las entrevistadas. No se encontraron diferencias en la distribución de las variables de predicción al 
comparar aquéllos que entregaron muestra con quienes no la entregaron. El porcentaje de desnutrición también fue igual para los dos grupos.

Nuestros resultados sugieren que, en niños menores de 2 años, es importante establecer de manera rutinaria el diagnóstico parasitológico para detectar oportunamente las infecciones por parásitos intestinales en el periodo del destete. Un documento de revisión de la Organización Mundial de la Salud pone en evidencia los efectos positivos y la seguridad del mebendazol y el albendazol sobre el desarrollo y la salud infantil en niños de 12 a 24 meses $(1,2)$. Los cambios en las condiciones de vida y de higiene, la educación de las madres, al igual que la desparasitación regular de poblaciones de alto riesgo mayores de 12 meses se proponen como estrategias para prevenir y disminuir la carga de enfermedad producida por el parasitismo intestinal en niños menores de 2 años. Sin embargo, la promoción de la lactancia materna prolongada sigue siendo la estrategia más costo-efectiva para estas comunidades.

\section{Agradecimientos}

A todo el personal del CEMPA.

\section{Conflictos de interés}

Ninguno.

\section{Financiación}

Este proyecto fue financiado por la Organización Panamericana de la Salud- Programa de subvenciones de tesis de salud pública. Referencia: HDP/HDR/RG-T/COL/3146 y por Colciencias, Convocatoria 2001, Plan Nacional de Ciencia y Tecnología de la Salud (código del proyecto: 1103-04-11985).

\section{Referencias}

1. Montresor A, Awasthi S, Crompton DW. Use of benzimidazoles in children younger than 24 months for the treatment of soil-transmitted helminthiasis. Acta Trop 2003;86:223-32.

2. World Health Organization. Report of the WHO informal consultation of the use of praziquatel during pregnancy/lactation and albendazole/mebendazole in children under 24 months. WHO/CDS/CPE/PVC/2002.4. Geneva: World Health Organization; 2002.
3. Corredor A, Arciniegas E, Hernández CA. Parasitismo Intestinal. Bogotá: Ministerio de Salud, Instituto Nacional de Salud; 2002. p.90.

4. Stephensen CB. Burden of infection on growth failure. J Nutr 1999;129(Suppl 2):534-8.

5. Crompton DW, Nesheim MC. Nutritional impact of intestinal helminthiasis during the human life cycle. Annu Rev Nutr 2002;22:35-59.

6. Stoltzfus RJ, Kvalsvig JD, Chwaya HM, Montresor A, Albonico M, Tielsch JM et al. Effects of iron supplementation and anthelmintic treatment on motor and language development of preschool children in Zanzibar: double blind, placebo controlled study. BMJ 2001;323:1389-93.

7. Stoltzfus RJ, Chway HM, Montresor A, Tielsch JM, Jape JK, Albonico $M$ et al. Low dose daily iron supplementation improves iron status and appetite but not anemia, whereas quarterly anthelminthic treatment improves growth, appetite and anemia in Zanzibari preschool children. J Nutr 2004;134:348-56.

8. Baqui AH, Black RE. Childhood infectious diseases and their contribution to undernutrition. In: Black RE, Fleischer K, editors. Public health issues in infant and health nutrition. Nestle Nutrition Workshops Series 2002;48:19-51.

9. Moore SR, Lima AA, Conaway MR, Schorling JB, Soares AM, Guerrant RL. Early childhood diarrhoea and helminthiases associate with long-term linear growth. Int J Epidemiol 2001;30:1457-64.

10. Bhan MK, Bahl R, Bhandari N. Infection: How important are its effects on child nutrition and growth? En: Martorell R, Hascke F, editors. Child nutrition and growth. Nestle Nutrition Workshops Series 2001;47:197-221.

11. Fraser D, Bilenko N, Deckelbaum RJ, Dagan R, ElOn J, Naggan L. Giardia lamblia carriage in Israeli Bedouin infants: risk factors and consequences. Clin Infect Dis 2000;30:419-24.

12. Lunn PG, Erinoso HO, Northrop-Clewes CA, Boyce SA. Giardia intestinalis is unlikely to be a major cause of the poor growth of rural Gambian infants. J Nutr 1999;129:872-7.

13. Muniz-Junqueira MI, Queiroz EF. Relationship between protein-energy malnutrition, vitamin $A$, and parasitoses in living in Brasilia. Rev Soc Bras Med Trop 2002;35:133-41.

14. Engle PL, Bentley M, Pelto G. The role of care in nutrition programmes: current research and a research agenda. Proc Nutr Soc 2000;59:25-35.

15. Fernandez ID, Himes JH, de Onis M. Prevalence of nutritional wasting in populations: building explanatory models using secondary data. Bull World Health Organization 2002;80:282-91. 
16 Smith H, Kaminsky R, Niwas S, Soto R, Jolly P. Prevalence and intensity of infections of Ascaris lumbricoides and Trichuris trichiura and associated socio-demographic variables in four rural Honduran communities. Mem Inst Oswaldo Cruz 2001;96:30314.

17 Saldiva SR, Silveira AS, Philippi ST, Torres DM, Mangini AC, Dias RM et al. Ascaris-Trichuris association and malnutrition in Brazilian children. Paediatr Perinat Epidemiol 1999;13:89-98.

18. Sackey ME, Weigel MM, Armijos RX. Predictors and nutritional consequences of intestinal parasitic infections in rural Ecuadorian children. J Trop Pediatr 2003;49:1723.

19. Mahmud MA, Chappell C, Hossain MM, Habib M, Dupont HL. Risk factors for development of first symptomatic Giardia infection among infants of a birth cohort in rural Egypt. Am J Trop Med Hyg 1995;53: 84-8.

20. Mahmud MA, Chappell CL, Hossain MM, Huang DB, Habib M, DuPont HL. Impact of breast-feeding on Giardia lamblia infections in Bilbeis, Egypt. Am J Trop Med Hyg 2001;65:257-60.

21. Costa-Macedo LM, Rey L. Maternal and child intestinal parasitism and breast-feeding. Rev Soc Bras Med Trop 2000;33:371-5.

22. Alvarado BE. Épidémiologie de la croissance infantile: Étude de déterminants sociaux et biologiques auprès d'enfants âgés de 6 à 18 mois en Colombie. En Médecine Sociale et Préventive. Montréal, QC: Université de Montréal: 2005.

23. Profamilia. Encuesta Nacional de Demografía y Salud. Región Pacifica. Santa Fe de Bogotá: Profamilia; 2000. p. $55-7$

24. Profamilia. Encuesta Nacional de Demografía y Salud. Santafe de Bogotá: Profamilia; 1995.

25. Burbano R. La construcción de la enfermedad en Guapi. Revista AntroPacifico 2003;1:149-60.

26. STATCALC. EPI-INFO: Software for Public Health: Center for Disease and Prevention, 2000.

27. Piwoz EG, Creed de Kanashiro H, López de Romana GL, Black RE, Brown KH. Feeding practices and growth among low-income Peruvian infants: a comparison of internationally-recommended definitions. Int J Epidemiol 1996;25:103-14.

28. Lohman T, Roche A, Fajardo L. Anthropometric standardization reference manual. Champaing, IL: Human Kinetics; 1990.

29. EPINUT. EPI-INFO: Software for Public Health. Atlanta. Centers for Disease and Prevention, 2000.

30. World Health Organization. Physical status: the use and interpretation of anthropometry. Technical Report Series. Geneva: World Health Organization; 1995.
31. World Health Organization. Medios auxiliares para el diagnostico de las parasitosis intestinales. Geneva: World Health Organization; 1994.

32. Duque S, Guerrero R, Nicholls RS, Lopez MC. Examen coproparasitológico en niños: comparación de resultados obtenidos por dos métodos en dos instituciones de Santa Fe de Bogotá. Biomédica 1994;14:39-47.

33. Espinal PA, López MC, de Durán G, Moncada LL, Delgado P. Parasitismo intestinal en la población infantil menor de 15 años en el servicio de consulta externa del Hospital de la Misericordia y evaluación de métodos para su diagnostico. Revista de la Facultad de Medicina, Universidad Nacional de Colombia 1998:46:66-74.

34. Rothman KJ, Greenland S. Approaches to statistical analysis: En: Rothman KJ, Greenland S, editors. Modern Epidemiology. Philadelphia: Lippincott Williams \& Wilkins; 1998. p.189-94.

35. Victora C, Huttly SR, Fuchs SC, Olinto MT. The role of conceptual framework in epidemiological analysis: A hierarchical approach. Int J Epidemiol 1997;26:224-7.

36. Trivino $\mathbf{X}$, Valenzuela $\mathbf{P}$, Torres $\mathbf{M}$, Anglony $\mathbf{M}$, Cordoba M. Enteroparásitos en lactantes de un centro universitario privado de salud en Santiago de Chile. Rev Chil Pediatr 2000;71:398-404.

37. Dominguez Vázquez A, Alzate Sánchez A. Nutritional status in children under 6 years of age and its association with malaria and parasitic intestinal diseases in Cordoba, Buenaventura, Colombia. Bol Chil Parasitol 1988;43:3-10.

38. Ordoñez LE, Angulo ES. Malnutrition and its association with intestinal parasitism among children from a village in the Colombian Amazonian region. Biomédica 2002;22:486-98.

39. Armar-Klemesu M, Ruel MT, Maxwell DG, Levin CE, Morris SS. Poor maternal schooling is the main constraint to good child care practices in Accra. J Nutr 2000;130:1597-607.

40. LeVine RA, LeVine SE, Rowe ML, Schnell-Anzola B. Maternal literacy and health behavior: a nepalese case study. Soc Sci Med 2004;58:863-77.

41. Gross R, Schell B, Molina MC, Leao MA, Strack U. The impact of improvement of water supply and sanitation facilities on diarrhea and intestinal parasites: a Brazilian experience with children in two low-income urban communities. Rev Saude Publica 1989;23:21420.

42. Fontbonne A, Freese-De-Carvalho E, Acioli MD, Sa GA, Cesse EA. Risk factors for multiple intestinal parasites in an indigenous community of the State of Pernambuco, Brazil. Cad Saude Publica 2001;17:36773.

43. Ludwig KM, Frei F, Alvares Filho F, Ribeiro-Paes JT. Correlation between sanitation conditions and 
intestinal parasitosis in the population of Assis, State of Sao Paulo. Rev Soc Bras Med Trop 1999;32:547-55.

44. Leon-Cavas N, Lutter C, Ross J, Martin L. Quantifying the benefits of breastfeeding: A summary of the evidence. Washington, DC: Panamerican Health Organization; 2002.

45. Gendrel D, Richard-Lenoble D, Kombila M, Baziomo JM, Gendrel C, Nardou M. Breast-feeding and intestinal parasites. Arch Fr Pediatr 1988;45:399-404.

46. Walterspiel JN, Morrow AL, Guerrero ML, RuizPalacios GM, Pickering LK. Secretory anti-Giardia lamblia antibodies in human milk: protective effect against diarrhea. Pediatrics 1994;93:28-31.

47. Costa-Macedo LM, Costa M, Almeida LM. Ascaris lumbricoides in infants: a population-based study in Rio de Janeiro, Brasil. Cad Saude Publica 1999;15:173-8.

48. Oberhelman RA, Guerrero ES, Fernández ML, Silio M, Mercado D, Comiskey N et al. Correlations between intestinal parasitosis, physical growth, and psychomotor development among infants and children from rural Nicaragua. Am J Trop Med Hyg 1998;58:470-5.

49. Dickson R, Awasthi S, Williamson P, Demellweek C, Garner P. Effects of treatment for intestinal helminth infection on growth and cognitive performance in children: systematic review of randomised trials. BMJ 2000;320:1697-701.

50. Stephenson LS, Latham MC, Ottesen EA. Malnutrition and parasitic infections. Parasitology 2000;121:s2338.

51. Walker SP, Grantham-McGregor SM, Himes JH, Powell CA. Relationships between wasting and linear growth in stunted children. Acta Paediatr 1996;85:666-9.

52. Newman RD, Moore SR, Lima AA, Nataro JP, Guerrant RL, Sears CL. A longitudinal study of Giardia lamblia infection in north-east Brazilian children. Trop Med Int Health $2001 ; 6: 624-34$.

\section{Anexo 1}

Lactancia exclusiva: si durante los primeros seis meses de vida el niño no recibió nada excepto la leche materna. Lactancia predominante: si el niño en los primeros seis meses de vida recibió leche materna más agua, jugos de frutas u otras bebidas no lácteas. Alimentación complementaria parcial: niños que recibieron, además de la leche materna, algún alimento sólido, leche en polvo reconstituida u otro tipo de leches o semisólidos. Sin lactancia/suspensión de lactancia materna: si no recibieron nunca la lactancia materna o si las madres suspendieron la lactancia en los primeros seis meses de vida (con otros líquidos o sólidos o sin ellos). 